\title{
Measurement of the cosmic ray spectrum with the Pierre Auger Observatory
}

\author{
Daniela Mockler ${ }^{1, a}$ for the Pierre Auger Observatory ${ }^{2, b}$ \\ ${ }^{1}$ Karlsruhe Institute of Technology, Institute of Experimental Particle Physics (ETP), Karlsruhe, Germany \\ ${ }^{2}$ Full author list and acknowledgments at: http://www.auger.org/archive/authors_2018_09.html
}

\begin{abstract}
The flux of ultra-high energy cosmic rays above $3 \times 10^{17} \mathrm{eV}$ has been measured with unprecedented precision at the Pierre Auger Observatory. The flux of the cosmic rays is determined by four different measurements. The surface detector array provides three data sets, two formed by dividing the data into two zenith angle ranges, and one obtained from a nested, denser detector array. The fourth measurement is obtained with the fluorescence detector. By combing all four data sets, the all-sky flux of cosmic rays is determined. The spectral features are discussed in detail and systematic uncertainties are addressed.
\end{abstract}

\section{Introduction}

The measurement of the energy spectrum of Ultra-High Energy Cosmic Rays (UHECRs) is key to answer the fundamental questions of the origin and propagation of these particles. The data taking of the Pierre Auger Observatory [1] started in 2004 and the high-quality data taken since then led to an unprecedented measurement of the UHECR spectrum. Two spectral features have been established undoubtedly: a flattening of the spectrum at an energy around $5 \times 10^{18} \mathrm{eV}$ (the ankle), and a steep flux suppression at the highest energies. The precise measurement of the flux, in combination with the mass composition and the distribution of the arrival directions of the particles over the sky, are important ingredients for astrophysical models describing the origin and the propagation of UHECRs.

The Pierre Auger Observatory exploits a hybrid detection technique to measure the extensive air showers induced by UHECRs impinging the Earth's atmosphere. The ground-based Surface Detector array (SD) records the lateral distribution of the secondary particles, while the Fluorescence Detector (FD) measures the calorimetric energy stored in the atmosphere. The SD is composed of 1600 waterCherenkov detectors, which are arranged in a hexagonal grid. With a spacing of $1500 \mathrm{~m}$, the SD covers an area of $3000 \mathrm{~km}^{2}$. The array is fully efficient for energies above $3 \times 10^{18} \mathrm{eV}$ [2]. A nested array with a reduced detector spacing of $750 \mathrm{~m}$ was built to increase the SD sensitivity towards lower energies. This array consists of 120 stations, of which 49 stations have been deployed in addition, and the remaining 71 stations are shared with the standard SD. The spanned area amounts to $27 \mathrm{~km}^{2}$ and the energy of full efficiency is lowered to $3 \times 10^{17} \mathrm{eV}$ [3]. The SD is sensitive to the electromagnetic and muonic shower component and the duty cycle is almost $100 \%[4,5]$. The atmosphere above the

\footnotetext{
a e-mail: daniela.mockler@kit.edu

be-mail: auger_spokespersons@fnal.gov
} 
SD is overlooked by 27 optical telescopes distributed into five buildings along the periphery of the array. The FD records the fluorescence light emitted by the nitrogen molecules that are excited by the secondary particles traversing through the atmosphere as well as the Cherenkov light induced by the secondaries. Therefore, the FD measures the longitudinal development of the air shower. The FD operation is only possible in clear and moonless nights, resulting in a duty cycle of about $13 \%[6,7]$.

We present the measurements of the flux of cosmic rays obtained with the four data sets and their combination to the all-sky flux spanning an energy range of almost three decades.

\section{Flux measurements with the SD and FD}

The reconstruction of the arrival direction and the core position with the SD array is based on the spatial and temporal combination of the individual station trigger times. The recorded signals are calibrated to the signal produced by a Vertical Equivalent Muon (VEM) [5, 8]. The attenuation in the atmosphere differs for the electromagnetic and muonic shower components, which necessitates different reconstruction methods for different zenith angle ranges. The SD data are divided into vertical events $\left(\theta<60^{\circ}\right)$ and inclined events $\left(60^{\circ} \leq \theta<80^{\circ}\right)$. The reconstruction of the vertical events is based on describing the recorded signals in the stations with a lateral distribution function. The expected signal $S\left(r_{\mathrm{opt}}\right)$ at an optimal distance $r_{\mathrm{opt}}$ from the shower axis gives the energy estimator for the SD measurements. The optimal distance depends on the grid spacing, resulting in the energy estimators $S(450)$ and $S(1000)$ for the $750 \mathrm{~m}$ and $1500 \mathrm{~m}$ arrays respectively. These energy estimators are zenith angle dependent. For a fixed energy, the observed signals at ground depend on the amount of traversed atmosphere due to the attenuation of the shower particles. This attenuation is corrected for by using a Constant Intensity Cut method [9]. Taking the median zenith angle of $38^{\circ}\left(35^{\circ}\right)$ for the $1500 \mathrm{~m}(750 \mathrm{~m})$ array, the expected signal $S\left(r_{\mathrm{opt}}\right)$ is corrected to a signal a shower would have produced had it arrived at $38^{\circ}\left(35^{\circ}\right)$. For the $750 \mathrm{~m}$ array, only events with zenith angles below $55^{\circ}$ are accepted, which results in a median angle of $35^{\circ}$. The reconstruction of inclined events differs due to the dominant muonic shower component. Due to the increased amount of traversed atmosphere by the shower, the electromagnetic component is largely absorbed [10]. The muons are deflected by the geomagnetic field and the cylindrical symmetry at ground, which is seen for vertical showers, is lost. The observed signal distributions at ground are fitted to muon density distributions predicted from simulated air showers. The energy estimator of inclined events is based on determining the relative muon content $N_{19}$ in the showers. The reference muon density corresponds to the one produced by a simulated proton shower with an energy of $10^{19} \mathrm{eV}$ [11]. The energy of the primary particle is inferred from $N_{19}$. For a high-quality SD event, the station with the highest signal has to be surrounded by a hexagon of six active stations. The calculation of the SD exposure is based on the determination of the geometrical aperture of the array for the respective zenith angle range and the observation time [2]. The exposure values up to $31 \mathrm{Dec} 2016$ for the different arrays are given in fig. 1a.

The energy calibration of the SD events is based on a data-driven approach by selecting a subset of high-quality events (called golden hybrid events) which have been measured by both the SD and FD [12]. The relation between the energy estimators $\hat{S}$ of the SD data sets, i.e. $S_{35}, S_{38}$, and $N_{19}$, and the energy $E_{\mathrm{FD}}$ measured by the telescopes is well-described by a power law $E_{\mathrm{FD}}=A \cdot \hat{S}^{B}$. The golden hybrid events are depicted in fig. $1 \mathrm{~b}$ for the three different SD data sets. Superimposed to the individual events are the calibration functions resulting from maximum-likelihood fits. The respective numbers of the golden hybrid events are given in addition. Due to the finite detector resolution, migration effects occur. Due to the steeply falling flux, more events migrate from lower energy bins towards higher energy bins. Therefore, the measured spectra are smeared with respect to the true one. To obtain the true spectra, a deconvolution is performed and the spectra are corrected by less than $10 \%$ in the energy range of interest. 
The four independent energy spectra derived from the data measured by Auger are shown in fig. 2a. As the SD spectra are calibrated with the energies measured by the FD, the SD energies share the uncertainty of the FD energy scale of $14 \%$. The hybrid sample is built from showers measured by the FD in coincidence with at least one triggered station of the SD. Combining the measurement of the FD with the timing information of the SD station allows for a very accurate determination of the shower geometry in the hybrid approach. The detector exposure is influenced by several factors concerning the atmospheric and data taking conditions. To mimic these conditions as closely as possible, the hybrid exposure is determined with the help of extensive Monte Carlo simulations that take into account the exact conditions of the observatory [7]. The derived energy spectrum starts at an energy of $10^{18} \mathrm{eV}$ and overlaps at lower energies with the spectrum obtained SD-750 $\mathrm{m}$ data and subsequently with the spectrum deduced from the SD-1500 $\mathrm{m}$ data.

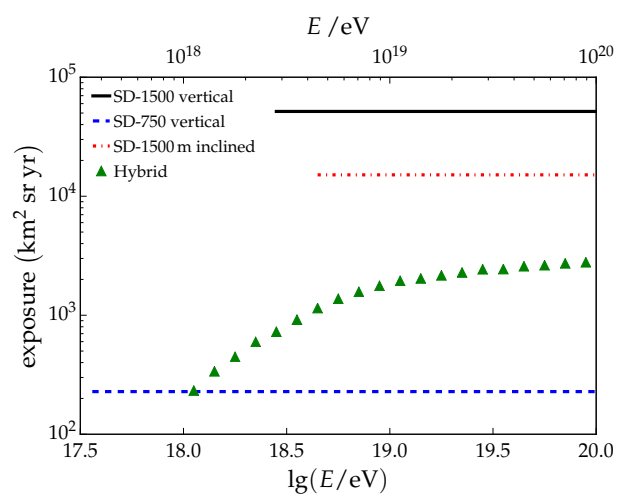

(a)

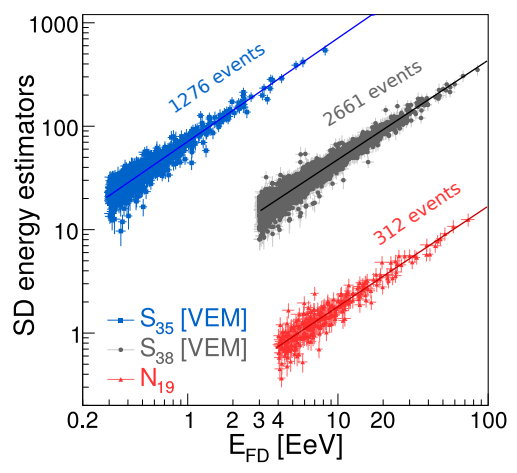

(b)

Figure 1: (a) Integrated exposure of the different detectors as a function of the energy. The SD exposure is flat above an energy corresponding to the full trigger efficiency. (b) Correlation between the SD energy estimates $S_{35}, S_{38}, N_{19}$ and the energy determined by the FD.

\section{Combined energy spectrum}

The combination is performed by means of a global maximum-likelihood fit. The flux normalizations are used as additional constraints to obtained the flux scaling factors that match the spectra: $(-0.8 \pm$ $0.2) \%$ for the SD-1500 vertical, $(-1 \pm 4) \%$ for the SD-750, $(5.4 \pm 0.7) \%$ for the SD-1500 horizontal and $(-6 \pm 2) \%$ for the hybrid. The spectral features are then derived by fitting the combined spectrum with the following function:

$$
J_{\text {unf }}(E)= \begin{cases}J_{0}\left(\frac{E}{E_{\text {ankle }}}\right)^{-\gamma_{1}} & E<E_{\text {ankle }} \\ J_{0}\left(\frac{E}{E_{\text {ankle }}}\right)^{-\gamma_{2}}\left[1+\left(\frac{E_{\text {ankle }}}{E_{\mathrm{s}}}\right)^{\Delta \gamma}\right]\left[1+\left(\frac{E}{E_{\mathrm{s}}}\right)^{\Delta \gamma}\right]^{-1} & E>E_{\text {ankle }}\end{cases}
$$

The combined spectrum, the fit, and the parameters are given in fig. $2 \mathrm{~b}$. The combined spectrum shows a flattening above the ankle, which is found at $E_{\text {ankle }}=(5.08 \pm 0.06$ (stat.) \pm 0.8 (syst. $\left.)\right) \times 10^{18} \mathrm{eV}$, while the suppression is obtained at $E_{\mathrm{s}}=(3.9 \pm 0.2$ (stat.) \pm 0.8 (syst. $\left.)\right) \times 10^{19} \mathrm{eV}$. The energy $E_{1 / 2}$ at which the integral spectrum drops by a factor of two below what would be the expected with no steepening is $E_{1 / 2}=(2.26 \pm 0.08$ (stat.) \pm 0.4 (syst.) $) \times 10^{19} \mathrm{eV}$. The spectral indexes are: $\gamma_{1}=3.293 \pm 0.002$ (stat.) \pm 0.05 (syst.), $\gamma_{2}=2.53 \pm 0.02$ (stat.) \pm 0.1 (syst.) while $\Delta \gamma=2.5 \pm 0.1$ (stat.) \pm 0.4 (syst.) [13]. 


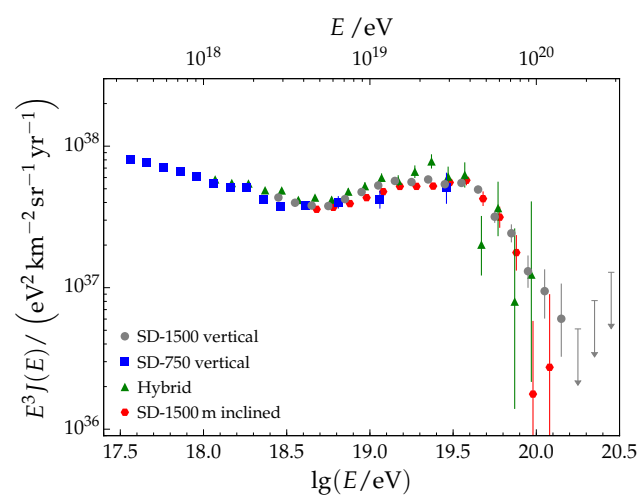

(a)

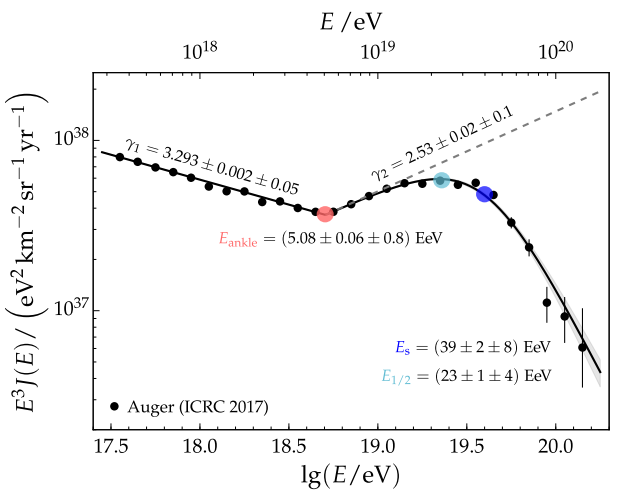

(b)

Figure 2: (a) Energy spectra obtained from the three SD data sets and the hybrid data. The systematic uncertainty on the energy scale, which is common to all of them, is $14 \%$. (b) Combined spectrum and fitted function. The optimal parameters are given in addition.

\section{References}

[1] A. Aab et al., Nuclear Instruments and Methods in Physics Research Section A: Accelerators, Spectrometers, Detectors and Associated Equipment A798, 172 (2015)

[2] J. Abraham et al. (Pierre Auger Collab.), Nucl. Instrum. Meth. A613, 29 (2010)

[3] D. Ravignani et al. (Pierre Auger Collab.), Measurement of the energy spectrum of cosmic rays above $3 \times 10^{17} \mathrm{eV}$ using the AMIGA $750 \mathrm{~m}$ surface detector array of the Pierre Auger Observatory, in Proc. 33rd Int. Cosmic Ray Conf. (Rio de Janeiro, Brazil, 2013)

[4] J. Abraham et al. (Pierre Auger Collab.), Nucl. Instrum. Meth. A523, 50 (2004)

[5] I. Allekotte et al. (Pierre Auger Collaboration), Nucl.Instrum.Meth. A586, 409 (2008)

[6] J. Abraham et al. (Pierre Auger Collab.), Nucl. Instrum. Meth. A620, 227 (2010)

[7] P. Abreu et al. (Pierre Auger Collaboration), Astropart. Phys. 34, 368 (2011)

[8] X. Bertou et al. (Pierre Auger Collaboration), Nucl. Instrum. Meth. A568, 839 (2006)

[9] J. Hersil, I. Escobar, D. Scott, G. Clark, S. Olbert, Phys. Rev. Lett. 6, 22 (1961)

[10] I. Valiño et al. (Pierre Auger Collab.), The electromagnetic component of inclined air showers at the Pierre Auger Observatory, in Proc. 31st Int. Cosmic Ray Conf. (Lódź, Poland, 2009)

[11] Rodriguez, G., A measurement of the muon number in showers using inclined events detected at the Pierre Auger Observatory, in European Physical Journal Web of Conferences (2013), Vol. 53 of European Physical Journal Web of Conferences, p. 07003

[12] R. Pesce et al. (Pierre Auger Collab.), Energy calibration of data recorded with the surface detectors of the Pierre Auger Observatory: an update, in Proc. 32nd Int. Cosmic Ray Conf. (Beijing, China, 2011)

[13] F. Fenu et al. (Pierre Auger Collab.), The cosmic ray energy spectrum measured using the Pierre Auger Observatory, in Proc. 35th Int. Cosmic Ray Conf. (Busan, South Korea, 2015) 